\title{
Allosteric Regulation of the Photosynthetic C4 Isoenzyme of Phosphoenolpyruvate Carboxylase: A Comparative Study Between Enzymes from Monocot and Dicot Plants ${ }^{1}$
}

\author{
Rodrigo Güémez-Toro, Carlos Mújica-Jiménez, and Rosario A. Muñoz-Clares*
}

Departamento de Bioquímica, Facultad de Química, Universidad Nacional Autónoma de México, México DF, 04510, México. 52 (55) 56225276; clares@unam.mx

Dedicated to Dr. Estela Sánchez de Jiménez by RAMC in gratitude for her suggestion for studying this enzyme, many years ago, and for her continuous support and example throughout the years

Received February 23, 2011; accepted June 8, 2011

\begin{abstract}
In the present study, we have investigated the complex allosteric regulation of the non-phosphorylated forms of the photosynthetic phosphoenolpyruvate carboxylase isoenzymes (PEPC-C4) from amaranth (AhPEPC-C4) and maize (ZmPEPC-C4) leaves. Previous studies showed that glycine (Gly) only activates PEPC-C4 from monocot plants, as maize, but not from dicot plants, as amaranth. Our initial velocity data confirm this, in spite that AhPEPC-C4 binds Gly with much higher affinity than ZmPEPC-C4. In AhPEPC-C4, the lack of Gly activation is overcome mainly by its higher affinity for the substrate phosphoenolpyruvate and its lower affinity for the inhibitor malate compared with ZmPEPC-C4. We have also explored the structural determinants of the differences in Gly activation by performing multiple alignments between the known monocot and dicot PEPC-C4 sequences and by modeling, in both the AhPEPC-C4 and ZmPEPC-C4 isoenzymes, the three-dimensional structure of the loop proposed as the Gly binding site, which was not observed in the crystal structure of the maize enzyme due to its high flexibility. The models suggest that conserved lysyl and aspartyl residues are important for binding to the activator molecule, and that a nearby non-conserved residue may be responsible for differences between the amaranth and maize enzymes in the loop conformation, which would account for the poorer affinity for Gly of the maize enzyme as well as for its higher degree of activation.

Key words: Zea mays L.; Amaranthus hypochondriacus L; C4 metabolism; allosteric activation; allosteric inhibition; amino acid sequence alignment; three-dimensional homology model.
\end{abstract}

\section{Introduction}

In leaves of $\mathrm{C} 4$ plants the initial reaction in the assimilation pathway of atmospheric $\mathrm{CO}_{2}$ is the essentially irreversible carboxylation of phosphoenolpyruvate (PEP) by phosphoenolpyruvate carboxylase (orthophosphate: oxaloacetate carboxy-lyase (phosphorylating), PEPC, EC 4.1.1.31) [1]. The importance of the PEPC-catalyzed reaction in the photosynthetic $\mathrm{CO}_{2}$ assimilation metabolism of $\mathrm{C} 4$ plants is underscored by the complex regulation of the activity of the PEPC-C4 isoenzyme. At physiological $\mathrm{pH}$, the maize leaf PEPC (ZmPEPC-C4) is activated allosterically by free PEP (fPEP) [2, 3], phosphorylated sugars [4,5], being glucose 6-phosphate (Glc6P) the strongest activator among them $[6,7,8]$, and by the neutral amino acids glycine (Gly) and serine (Ser) [6,9], whereas it is inhibited by dicarboxylic acids such as malate and aspartate [10]. The enzyme is
Resumen. En este trabajo, investigamos la compleja regulación alostérica de la formas no fosforiladas de las isoenzimas fotosintéticas de la fosfoenolpiruvato carboxilasa (PEPC-C4) de hojas de amaranto (AhPEPC-C4) y de maíz (ZmPEPC-C4). Estudios previos mostraron que glicina (Gly) sólo activa a las isoenzimas PEPC-C4 de plantas monocotiledóneas, como el maíz, y no a las de plantas dicotiledóneas, como el amaranto. Nuestros estudios de velocidad inicial confirman estos resultados, a pesar de que la AhPEPC-C4 une a Gly mejor que la ZmPEPC-C4. La falta de activación por Gly en la AhPEPC-C4 se compensa principalmente por su mayor afinidad por el sustrato fosfoenolpiruvato y su menor afinidad por el inhibidor malato. También exploramos las bases estructurales de las diferencias en la activación por Gly haciendo alineamientos múltiples de las secuencias conocidas de PEPC-C4 de plantas mono y dicotiledóneas, así como modelando, tanto en la AhPEPC-C4 como en la ZmPEPC-C4, el asa propuesta como el sitio de unión de Gly, que no se observó en la estructura cristalográfica de la enzima de maíz debido a su alta flexibilidad. Los modelos sugieren que residuos conservados de lisina y aspártico son importantes para la unión del activador, y que un residuo cercano no conservado puede ser responsable de diferencias entre las enzimas de amaranto y de maíz en la conformación de este asa, lo que daría cuenta de la menor afinidad por Gly de la enzima de maíz así como de su mayor grado de activación.

Palabras clave: Zea mays L.; Amaranthus hypochondriacus L; metabolismo $\mathrm{C} 4$; activación alostérica; inhibición alostérica; alineamiento múltiple de secuencias de aminoácidos; modelo tridimensional por homología.

additionally regulated by phosphorylation on an N-terminal Ser serine residue [11], which causes a decrease in affinity for the dicarboxylic acids $[11,12]$ and an increase in affinity for PEP [13] or for the complex MgPEP [3]. It has been reasoned [14] that the main features of the kinetics of ZmPEPC-C4 would lead to an enzyme mostly inactive at the physiological concentrations of the substrate and inhibitor if the concentrations of allosteric activators were low.

The two kinds of allosteric activators of ZmPEPC-C4 produce a faster $\mathrm{C} 4$ cycle as a result of PEPC-C4 activation (Scheme 1). But they are by no means redundant. They allow for the fine-tuning of the fluxes between the two metabolic pathways involved in $\mathrm{CO}_{2}$ assimilation in $\mathrm{C} 4$ plants: the $\mathrm{C} 4$ and the Calvin cycles. The concentration of phosphorylated sugars increases when the Calvin cycle is active. Neutral amino acids concentrations, particularly that of Gly, increase under 


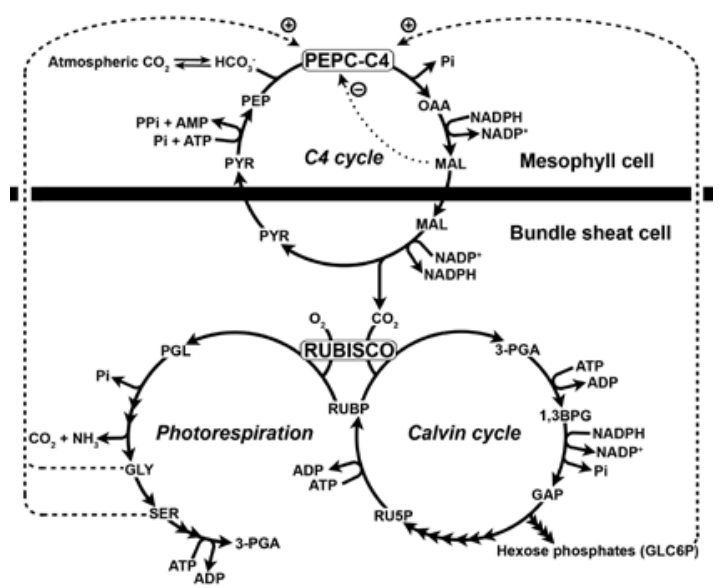

Scheme 1. Allosteric regulation of ZmPEPC-C4. The two different kinds of allosteric activators (neutral amino acids and phosphorylated sugars) and the inhibitor act as metabolic signals that allow the finetuning of the fluxes between the two metabolic pathways involved in $\mathrm{CO}_{2}$ assimilation in $\mathrm{C} 4$ plants: the $\mathrm{C} 4$ and the Calvin cycles. Phosphorylated sugars, whose concentration increases when the Calvin cycle is active, activate ZmPEPC-C4. Neutral amino acids, whose concentrations increase under photorespiration conditions, also activate $\mathrm{ZmPEPC}-\mathrm{C} 4$. Malate produces a feedback inhibition of ZmPEPC-C4 when its concentration increases because the Calvin cycle is not active. Abbreviations used: 1,3BPG, 1,3-bisphosphoglycerate; GAP, glyceraldehyde-3-phosphate; GLC6P, glucose-6-phosphate; GLY, glycine; MAL, malate; OAA, oxaloacetate; PEP, phosphoenolpyruvate; PEPC$\mathrm{C} 4, \mathrm{C} 4$ isoform of phosphoenolpyruvate carboxylase; PGL, phosphoglycolate; 3-PGA, 3-phosphoglycerate; Pi, inorganic phosphate; PPi, pyrophosphate; PYR, pyruvate; RUBISCO, ribulose-1,5-bisphophate carboxylase/oxygenase; RU5P, ribulose-5-phosphate; RUBP, ribulose-1,5-bisphosphate; SER, serine.

photorespiration conditions [15]. Therefore, the two kinds of activators act as metabolic signals that indicate the necessity of increasing the flux through the $\mathrm{C} 4$ cycle, in order to keep pace with the flux rate of the Calvin cycle in the case of Glc6P, or to increase the supply of $\mathrm{CO}_{2}$ to the bundle sheath cells to prevent photorespiration, in the case of Gly.

The binding of phosphorylated sugars and neutral amino acid to their respective allosteric sites affects the inhibition of the ZmPEPC-C4 enzyme by malate in quite different ways. While Glc6P is unable to revert the inhibition caused by a physiological concentration of malate, Gly can produce an enzyme almost as active than that in the absence of the inhibitor [14]. The kinetic differences between the allosteric activators acquire special relevance under conditions close to those prevailing under illumination, i.e., high malate concentrations and low $\mathrm{CO}_{2}$, when the degree of activation of the enzyme brought about by Glc6P is much lower than that brought about by neutral amino acids [14]. Activation by Glc6P could be important during the night or at the onset of illumination before the buildup of malate that takes place during the first hour after illumination [16]. Once the levels of malate are high, saturation of the Glc6P allosteric site would give only a marginal advantage. On the other hand, the allosteric activation by neutral amino acids is crucial for achieving appreciable levels of ZmPEPC$\mathrm{C} 4$ activity at low $\mathrm{CO}_{2}$, i.e., under photorespiratory conditions, when the concentration of this amino acid reaches significant levels (30-40 mM) [15]. Photorespiration surely follows the buildup of malate during the day because of a decrease of the C4 cycle flux, a decrease due to both PEPC inhibition by the increased malate concentration and depletion of the available $\mathrm{CO}_{2}$ by a very active Calvin cycle. Activation by Gly helps in increasing the flux through the $\mathrm{C} 4$ pathway by effectively counteracting the inhibitory effects of malate, and, therefore, it helps in increasing the concentrations of $\mathrm{CO}_{2}$ in the bundle sheet cells thus overcoming photorespiration. Photorespiration would perdure if Glc6P were the only ZmPEPC-C4 activator, given the inability of this activator to counteract the inhibition by malate of this enzyme.

Given its important physiological role, it is rather surprising that activation by Gly and other neutral amino acids had been only found in PEPC-C4 isoenzymes from monocot plants, while those from dicot plants are insensitive to these compounds [9]. One possible explanation for these findings is that the PEPC-C4 isoenzymes from dicots have different kinetic properties that those from monocots, so that activation by neutral amino acids is not needed for an efficient $\mathrm{CO}_{2}$ assimilation. For instance, the dicot enzymes may respond to malate and/or Glc6P differently than the ones from monocots. To find out if this is the case, in this work we carried out a comparative kinetic study of the allosteric regulation of the PEPC-C4 isoenzymes from Amaranthus hypochondriacus (AhPEPC-C4) and that from Zea mays (ZmPEPC-C4), which are dicot and monocot $\mathrm{C} 4$ plants respectively, under near physiological conditions. I addtion, to get insight into the possible structural bases of the insensitivity of the dicot isoenzymes to neutral amino acids, we carried out multiple alignments of the PEPC$\mathrm{C} 4$ amino acid sequences available in data banks, and modeled the AhPEPC-C4 and ZmPEPC-C4 loops that form part of the putative Gly binding site, a loop that was not observed in the maize enzyme crystal [17].

\section{Results and discussion}

\section{Kinetic properties of AhPEPC-C4 and ZmPEPC-C4}

To understand how AhPEPC-C4 and ZmPEPC-C4 respond to changes in their environment, in the present study we investigated their kinetic features at conditions close to those existing in vivo. The amount of free $\mathrm{Mg}^{2+}\left(\mathrm{fMg}^{2+}\right)$ estimated to be in the plant cytosol is only $0.4 \mathrm{mM}$, and this concentration is not believed to drastically change under any plausible physiological condition [18]. The concentration of bicarbonate in the cytosol of maize mesophyll cells under air and normal illumination conditions has been estimated to be $77 \mu \mathrm{M}$ [19] and the reported $\mathrm{pH}$ of the cytosol of these cells to be 7.3 [20]. The bicarbonate concentration in an assay medium in contact with air at $\mathrm{pH} 7.3$ when no bicarbonate has been added is $0.1 \mathrm{mM}$, a concentration slightly higher than the physiological intracellular concentration of mesophyll cells. Because of the above, the kinetic parameters for AhPEPC-C4 and ZmPEPC-C4 were 
determined at $\mathrm{pH}$ 7.3, $0.4 \mathrm{mM} \mathrm{fMg}{ }^{2+}$ and $0.1 \mathrm{mM}$ bicarbonate. Also, because regulation of PEPC activity by metabolite effectors is mostly exerted at subsaturating concentrations of substrate [21], in the studies with the allosteric effectors we used a fixed total PEP (tPEP) concentration of only $0.2 \mathrm{mM}$, which is a concentration believed to exist in mesophyl cells under dark conditions [22].

The results of these kinetic experiments are shown in Figure 1 and summarized in Table 1. Saturation of PEPC-C4 by its substrate PEP (Fig. 1A) was cooperative in both enzymes, with similar Hill coefficients $(h)$, but the concentration of substrate that gives half-maximum velocity $\left(S_{0.5}\right)$ for AhPEPC-C4 was $\approx$ 3-times lower than the value for ZmPEPC-C4, resulting in an activity of the amaranth enzyme significantly higher than that of the maize enzyme in the tPEP concentration range of 0.1 to $3 \mathrm{mM}$ (Fig. 1A, shaded area). These two limiting concentrations of tPEP are close to those existing in the cytosol of the mesophyll cells during the dark and light periods, respectively $[22,23]$.

Given the competitive nature of the inhibition of PEPC$\mathrm{C} 4$ enzymes by dicarboxylic acids $[13,14,24]$, the increase in the affinity of the AhPEPC-C4 enzyme for the substrate PEP has as a consequence that its sensitivity to malate inhibition, measured by the concentration of inhibitor that gives halfmaximum inhibition at fixed concentrations of substrates $\left(I_{50}\right)$, was about 3-times lower than that of the ZmPEPC-C4 enzyme (Table 1 and Figure 1B). In both enzymes, binding of malate is non-cooperative, as found in previous studies on ZmPEPCC4 [14, 25-27].

Contrary to the response to malate, but again consistent with the differences between the two enzymes in PEP affinity given the synergy between the binding of substrate and the binding of the activators in PEPC enzymes [3, 8], the affinity of the AhPEPC-C4 enzyme to Glc6P, measured by the concentration of activator that gives half-maximum activation at fixed concentrations of substrates $\left(A_{0.5}\right)$, was 9-times higher than that of the ZmPEPC-C4 enzyme (Table 1 and Figure 1C). Glc6P binds cooperatively to both enzymes, with $h$ values close to 2.0. Even though the maximum activation $\left(A c t_{\max }\right)$ was more than two-times higher in the maize enzyme, the degree of activation achieved at low Glc6P concentrations was significantly higher in the amaranth than in the maize enzyme.

Regarding the activation by Gly, the affinity of AhPEPC$\mathrm{C} 4$ was 7-times higher than that of ZmPEPC-C4, again consistent with the higher affinity for $\mathrm{PPEP}$ of the amaranth enzyme, but the maximum activation was almost 90-times lower, making Gly a very poor activator of the amaranth enzyme compared with the maize one (Table 1 and Figure 1D). Interestingly, Gly binds non-cooperatively to AhPEPC-C4, whereas a Hill coefficient close to 2.0 was observed in ZmPEPC-C4. This noncooperative binding is consistent with the lack of an allosteric transition triggered by the binding of Gly in AhPEPC-C4, as indicated by the very low degree of activation observed.

Taken together, these results confirm that in ZmPEPC-C4 the activation by Gly is physiologically more relevant than the activation by Glc6P, even at the low concentrations of substrate
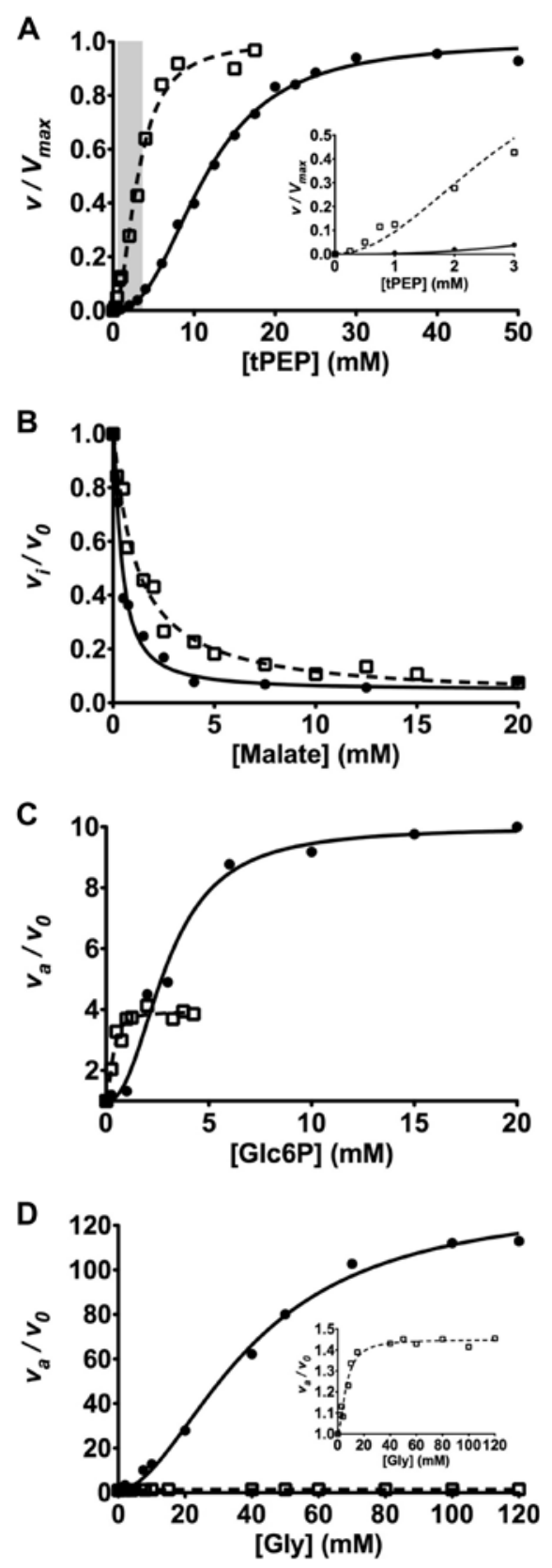

Fig. 1. Kinetics of AhPEPC-C4 (open squares) and ZmPEPC-C4 (solid circles) at $\mathrm{pH} 7.3,0.4 \mathrm{mM} \mathrm{fMg}^{2+}$ and $0.1 \mathrm{mM}$ bicarbonate. (A) Saturation by $\mathrm{tPEP}$. In the concentration range of tPEP used in these experiments $(0.03$ to $18 \mathrm{mM}$ for AhPEPC-C4 and 0.03 to $50 \mathrm{mM}$ for $\mathrm{ZmPEPC}-\mathrm{C} 4)$, MgPEP concentrations ranged from 0.002 to 1.21 $\mathrm{mM}$ for AhPEPC-C4, and from 0.002 to $3.36 \mathrm{mM}$ for ZmPEPC-C4, tPEP (trianionic form) concentrations ranged from 0.028 to 16.79 $\mathrm{mM}$ for AhPEPC-C4 and from 0.028 to $46.64 \mathrm{mM}$ for ZmPEPC-C4. The shaded area corresponds to the estimated physiological range of tPEP concentration existing under dark and light conditions $(0.1$ to 3 $\mathrm{mM}$, respectively). The insert shows an enlargement of the enzyme activity response to tPEP concentrations in this physiological range. (B) Saturation by malate. (C) Saturation by Glc6P. (D) Saturation by Gly. Insert: Enlargement of the Gly saturation data of AhPEPC-C4 that cannot be fully appreciated in the main figure. In panels (B), (C) and (D) the concentration of tPEP was $0.2 \mathrm{mM}$, corresponding to 0.013 $\mathrm{mM} \mathrm{MgPEP}$ and $0.187 \mathrm{mM}$ fPEP. The points are the experimental data; the lines are the result of the best fit of the experimental data to equations 1,2 , or 3 , as appropriate. 
Table 1. Apparent kinetic parameters ${ }^{\mathrm{a}}$ of AhPEPC-C4 and $\mathrm{ZmPEPC}-\mathrm{C} 4$ at $\mathrm{pH} 7.3,0.4 \mathrm{mM} \mathrm{fMg}^{2+}$ and $0.1 \mathrm{mM}$ bicarbonate.

\begin{tabular}{lcc}
\hline \multicolumn{1}{c}{ Parameter } & AhPEPC-C4 & ZmPEPC-C4 \\
\hline Saturation by tPEP & & \\
$S_{0.5}(\mathrm{mM})$ & $3.0 \pm 0.1$ & $11.3 \pm 0.1$ \\
$h$ & $2.0 \pm 0.2$ & $2.5 \pm 0.1$ \\
Saturation by malate $^{\mathrm{b}}$ & & \\
$I_{50}(\mathrm{mM})$ & $1.24 \pm 0.08$ & $0.43 \pm 0.04$ \\
Saturation by Glc6P & & \\
Act $_{\text {max }}$ & $3.9 \pm 0.2$ & $10.1 \pm 0.4$ \\
$A_{0.5}(\mathrm{mM})$ & $0.3 \pm 0.0$ & $3.0 \pm 0.3$ \\
$h$ & $1.9 \pm 0.6$ & $2.2 \pm 0.4$ \\
Saturation by Gly & & \\
$A c t_{\text {max }}$ & $1.5 \pm 0.0$ & $128 \pm 7$ \\
$A_{0.5}(\mathrm{mM})$ & $5.8 \pm 0.9$ & $41.6 \pm 3.2$ \\
$h$ & $1.3 \pm 0.2$ & $2.2 \pm 0.3$ \\
\hline
\end{tabular}

${ }^{a}$ Values \pm SD were estimated by the best fit of the initial velocity data to equation 1 for saturation by tPEP, equation 2 for saturation by Glc6P or Gly, or equation 3 for saturation by malate. ${ }^{\mathrm{b}}$ The apparent kinetic parameters were determined at $0.2 \mathrm{mM}$ tPEP. Other conditions are given in the Experimental section.

used in this study. The activation by Glc6P may, however, play an important role increasing the flux of the $\mathrm{C} 4$ cycle at the onset of the light conditions, as mentioned above.

\section{Effects of the inhibitor malate on the kinetics properties of AhPEPC-C4 and ZmPEPC-C4}

When $20 \mathrm{mM}$ malate was added to the assay media, the kinetic differences between AhPEPC-C4 and ZmPEPC-C4 were accentuated. Figure $2 \mathrm{~A}$ and Table 2 show that the kinetics of saturation by tPEP of ZmPEPC-C4 were highly cooperative, with a $h$ value close to the maximum attainable by a tetrameric enzyme, whereas only a small increase in the $h$ value of the AhPEPC-C4, compared with that estimated in the absence of malate, was observed. The differences between the two enzymes in the degree of cooperativity in the binding of PEP in the presence of a high malate concentration are in full agreement with their differences in malate affinity. Although the $S_{0.5}$ (tPEP) value of ZmPEPC-C4 was 4-times higher than that of AhPEPC-C4, similar to that found in the absence of malate, the higher cooperativity of $\mathrm{ZmPEPC}-\mathrm{C} 4$ results in even bigger differences between the two enzymes in their activities within the physiological concentration range of tPEP than those found in the absence of the inhibitor (inset Fig. 2A).

It has been reported that Glc6P effectively overcomes the inhibition by malate of ZmPEPC-C4 [11, 12], but these studies were performed at non-physiological, very high bicarbonate and total $\mathrm{Mg}^{2+}$ concentrations. When near physiological concentrations were used, Glc6P was very ineffective in overcoming malate inhibition [14]. Gly has been found to be much more effective than Glc6P in this respect under conditions close to
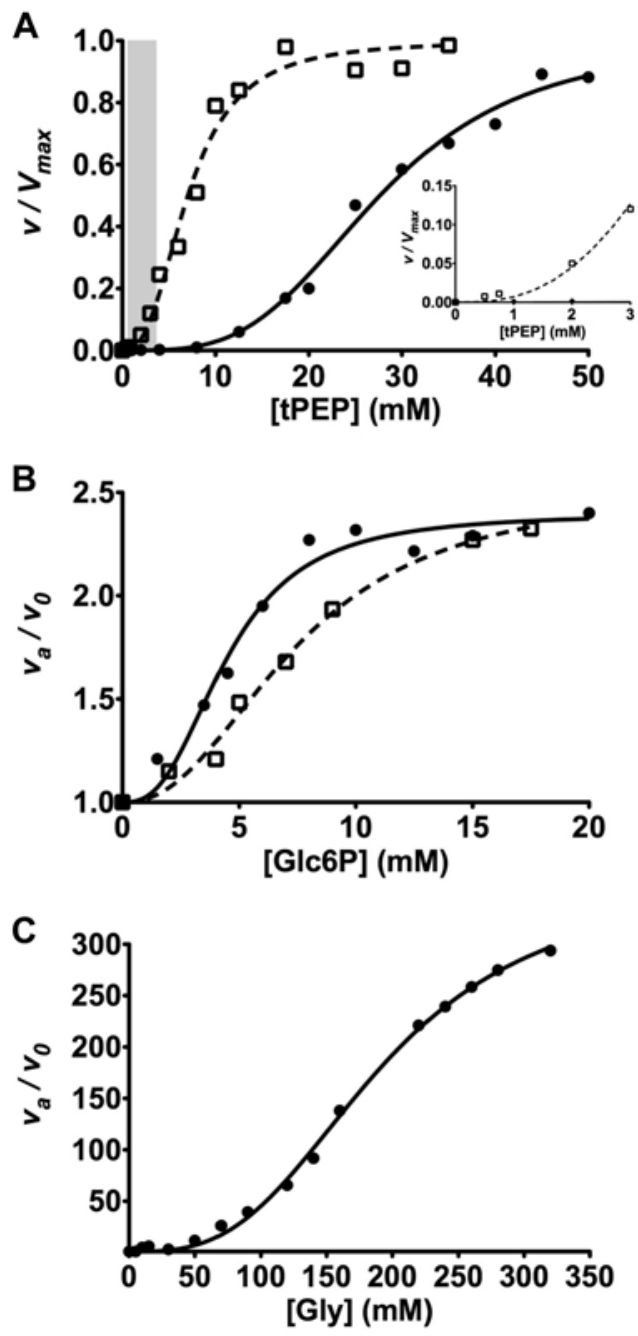

Fig. 2. Effect of $20 \mathrm{mM}$ L-malate on the kinetics of AhPEPC-C4 (open squares) and ZmPEPC-C4 (solid circles) at $\mathrm{pH} 7.3,0.4 \mathrm{mM} \mathrm{fMg}^{2+}$ and $0.1 \mathrm{mM}$ bicarbonate. (A) Saturation by tPEP. The shaded area corresponds to the estimated physiological range of tPEP concentration $(0.1$ to $3 \mathrm{mM}$ ). The insert shows an enlargement of the activity response to tPEP concentrations in this physiological range. (B) Saturation by Glc6P. (C) Saturation by Gly. Other experimental conditions are as in Figure 1. The points are the experimental data; the lines are the result of the best fit of the experimental data to equations 1 or 2 , as appropriate.

those existing in vivo during the light period [14]. We tested now the relative contribution of the two kinds of activators in relieving malate inhibition of the two $\mathrm{C} 4$ isoenzymes at the tPEP concentration existing during the night, $0.2 \mathrm{mM}$, but in the presence of $20 \mathrm{mM}$ malate. In AhPEPC-C4, a high malate concentration did not significantly affect the maximum activation achieved by a saturating concentration of Glc6P, which has a value $\approx 3$ similar to the value observed in the absence of malate, but malate did increase the $A_{0.5}$ for Glc6P $\approx 25$-times (Fig. 2B and Table 2). These results indicate that the binding of malate and that of Glc6P to the amaranth enzyme are competitive. On the contrary, in $\mathrm{ZmPEPC}-\mathrm{C} 4$ the kinetics of saturation by Glc6P were not significantly affected by malate, as indicated 
Table 2. Apparent kinetic parameters ${ }^{\mathrm{a}}$ of AhPEPC-C4 and $\mathrm{ZmPEPC}-\mathrm{C} 4$ at $\mathrm{pH} 7.3,0.4 \mathrm{mM} \mathrm{fMg}^{2+}, 0.1 \mathrm{mM}$ bicarbonate, and 20 $\mathrm{mM}$ malate.

\begin{tabular}{lcc}
\hline \multicolumn{1}{c}{ Parameter } & AhPEPC-C4 & ZmPEPC-C4 \\
\hline Saturation by tPEP & & \\
$S_{0.5}(\mathrm{mM})$ & $6.8 \pm 0.3$ & $27.4 \pm 0.4$ \\
$h$ & $2.44 \pm 0.2$ & $3.5 \pm 0.2$ \\
Saturation by Glc6P & & \\
$A c t_{\max }$ & $2.6 \pm 0.2$ & $2.4 \pm 0.1$ \\
$A_{0.5}(\mathrm{mM})$ & $7.9 \pm 1.4$ & $4.5 \pm 0.4$ \\
$h$ & $2.06 \pm 0.4$ & $2.5 \pm 0.6$ \\
Saturation by Gly & & \\
$A c t_{\text {max }}$ & nd & $367 \pm 17$ \\
$A_{0.5}(\mathrm{mM})$ & nd & $193 \pm 8$ \\
$h$ & nd & $3.0 \pm 0.2$ \\
\hline
\end{tabular}

${ }^{\text {aValues }} \pm$ SD were estimated by the best fit of the initial velocity data to equations 1 for saturation by tPEP, or 2 for saturation by Glc6P or Gly. ${ }^{\mathrm{b}}$ The apparent kinetic parameters were determined at $0.2 \mathrm{mM}$ tPEP. Other conditions are given in the Experimental section. ${ }^{\mathrm{c}}$ nd, Not determined due to lack of response of the enyme to the activator.

by the similar values of $A_{0.5}$ and Hill coefficient values obtained in the absence and presence of the inhibitor. These findings suggest that the binding of Glc6P is not affected by the binding of the inhibitor to this enzyme. In the maize isoenzyme, there was a 4-times decrease in the maximum activation achieved by Glc6P when in presence of malate, again indicating the low effectivity of this activator in relieving malate inhibition, as found in earlier studies under other conditions [14]. This is consistent with a lack of effect of malate on the binding of Glc6P and, reciprocally, a lack of effect of Glc6P on the binding of malate. Considering our present results, the kinetics of saturation by Glc6P of the two kinds of PEPC-C4 isoenzymes become similar in the presence of $20 \mathrm{mM}$ malate. Therefore, under our experimental conditions Glc6P is no more effective in counteracting malate inhibition of the amaranth than of the maize enzyme (Fig. 2B and Table 2).

The kinetics of saturation of the ZmPEPC-C4 enzyme with Gly in the presence of $20 \mathrm{mM}$ malate showed a decrease in the apparent affinity for the activator, as indicated by the $A_{0.5}$ value which was 5-fold higher than that estimated in the absence of the inhibitor. This is consistent with competition between inhibitor and activator for their binding to the enzyme. Moreover, different from Glc6P, Gly effectively relieved the inhibition in the monocot enzyme achieving a maximum activation 3-times higher than that in the absence of malate. The maximum activity estimated under the conditions of our assays at saturating Glc6P is only $\approx 0.65 \%$ of the maximum activity estimated at saturating Gly, thus confirming previous results obtained with the phosphorylated ZmPEPC-C4 at $3 \mathrm{mM}$ tPEP [14]. Gly did not activate AhPEPC-C4 in the presence of malate, so that saturation by Gly of this enzyme could not be determined under these conditions. These results show that Gly is not an activator of the dicot enzyme either in the absence or in the presence of the inhibitor malate.

\section{Multiple alignment of PEPC-C4 amino acid sequences}

Assuming that most PEPC-C4 isoenzymes from monocots plants are sensitive to neutral amino acid activation and that most of those from dicot plants are not, we thought of interest to investigate those amino acids positions of the putative allosteric site for neutral amino acid that show non-conservative substitutions between the two groups. In the protein database of the National Center for Biotechnology Information (NCBI), a total of 13 non-redundant protein sequences (allelic forms excluded) were identified as plant PEPC-C4 isoforms and used for sequence alignments, which were carried out as described in the Material and methods section. Six of these sequences are from monocot plants and the other seven from dicot plants. The overall identity among monocot isoenzymes ranged from 80 to $99.3 \%$ and for dicot isoenzymes from 74.9 to $87.8 \%$. Between monocot and dicots PEPC-C4 isoenzymes the identity was in the range from 60.0 to $79.2 \%$.

Shown in Figure 3 is the alignment of the portion of the carboxy-terminus region that constitutes a loop proposed to form part of the neutral amino acid binding site [28]. The eight amino acid residues that correspond to the portion of the loop not observed in the ZmPEPC-C4 crystal structure (residues 929-935 ZmPEPC-C4 numbering and 922-929 AhPEPC-C4 numbering) [17], probably due to its high conformational flex-

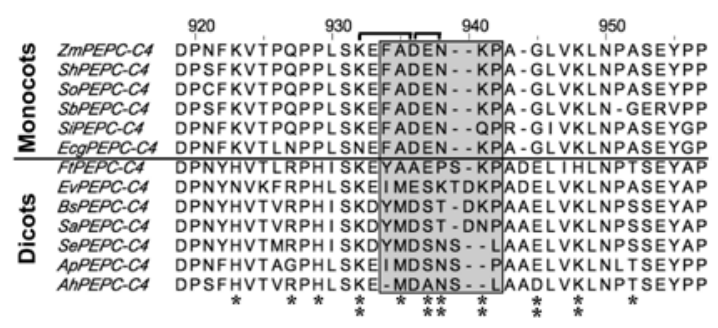

Fig. 3. Sequence alignments of PEPC-C4 isoforms. Only the residues forming part of the loop that forms part of the putative Gly-binding site are shown. The alignment positions marked with one asterisk are conserved or have conservative substitutions within each group of monocot or dicot isoenzymes but differ from one group to the other. Those positions marked with two asterisks have been changed by site directed mutagenesis in ZmPEPC-C4 [28]. Alignment positions 934 to 942 (residues 929-935 ZmPEPC-C4 numbering, or 922-929 AhPEPC-C4 numbering), enclosed in the shaded area, correspond to residues that form part of the flexible region of the loop not observed in the $\mathrm{ZmPEPC}-\mathrm{C} 4$ crystal structure [17]. The two main interactions observed in the homology model of AhPEPC-C4 (see main text) are indicated with solid lines at the top of the figure. The PEPC-C4 sequences used in the alignments are from the following plants: $\mathrm{Zm}-$ PEPC-C4, Zea mays; ShPEPC-C4, Saccharum hybrid; SoPEPC-C4, Saccharum officinarum; SbPEPC-C4, Sorghum bicolor; SiPEPC-C4 Setaria italica; EcgPEPC-C4, Echinochloa crus-galli; FtPEPC-C4, Flaveria trinervia; EvPEPC-C4, Eleocharis vivipara; BsPEPC-C4 Bienertia sinuspersici; SaPEPC-C4 Suaeda aralocaspica; SePEPCC4, Suaeda eltonica; ApPEPC-C4, Alternanthera pungen; AhPEPC$\mathrm{C} 4$, Amaranthus hypochondriacus. 
ibility, are enclosed in a shadowed box. In this loop there are several amino acid residues that are conserved, or with conservative substitutions, within each group of monocots or dicots enzymes, but that differ from one group to the other (marked with an asterisk in Figure 3). These residues may cause the differences between the two kinds of PEPC-C4 isoenzymes regarding activation by neutral amino acids. The residues corresponding to positions 932, 937, 938, 941, 945 and 948 of the alignment, marked in Figure 3 with two asterisks, have been already studied in ZmPEPC-C4 by site directed mutagenesis [28]. From these studies it was concluded that two of them (alignment positions 941 and 945, corresponding to K934 and G937 respectively in ZmPEPC-C4, and to a gap and D931 respectively in AhPEPC-C4) are important for Gly activation [28]. In addition, the conserved residue in the alignment position 932, corresponding to K927 and K921 in ZmPEPC-C4 and AhPEPC-C4 respectively, was found to also be important for Gly activation in the maize enzyme [28]. This lysyl residue may be involved in the binding of the carboxylic group of the amino acid in both kinds of isoenzymes, since PEPC-C4 from dicot plants are also able to bind these allosteric effectors, as our results with the amaranth enzyme indicate (this work).

\section{Three-dimensional homology models of AhPEPC-C4 and ZmPEPC-C4}

To date, the three-dimensional structures of PEPC enzymes known are those of the E. coli enzyme (EcPEPC) $[29,30]$ and of the ZmPEPC-C4 [17]. These crystal structures showed the active site with a $\mathrm{Mn}^{2+}$ ion and a PEP analog bound (in EcPEPC, Protein Data Bank (PDB) accesion codes 1QB4 and 1JQN), the malate binding site with an aspartate molecule bound (in EcPEPC, PDB accesion codes 1FIY, 1QB4, and 1JQN), and the Glc6P binding site with a sulphate ion bound (in ZmPEPC-C4, PDB accesion code 1JQO). The neutral amino acid binding site is not yet known because no structure with this kind of ligand has been determined so far.

In an attempt to find out whether any of the residues found in the alignment discussed above is at a position potentially critical for neutral amino acid activation, we constructed threedimensional homology models of the amaranth and maize enzymes (Figure 4) based on the published three-dimensional structure of ZmPEPC-C4 (PDB accesion code 1JQO). According to the AhPEPC-C4 model, the enzyme can be described as a dimer of dimers and has a general structural organization similar to that of ZmPEPC-C4, which is not surprising given the high degree of identity between them $(76.6 \%)$. But our interest was to model those parts of the ZmPEPC-C4 structure that were not observed in the crystal because of their high flexibility. In the homology models of both enzymes, these parts are forming loops, as expected. Of particular interest to us is the loop analyzed in the sequence alignments of Figure 3. Interestingly, the AhPEPC-C4 model shows a saline bridge between residues K921 and D924 (AhPEPC-C4 numbering), as well as a hydrogen bond between one of the oxygen atoms of the sidechain carboxyl group of $\mathrm{D} 924$ and the $\mathrm{NH}_{2}$ of the amide group

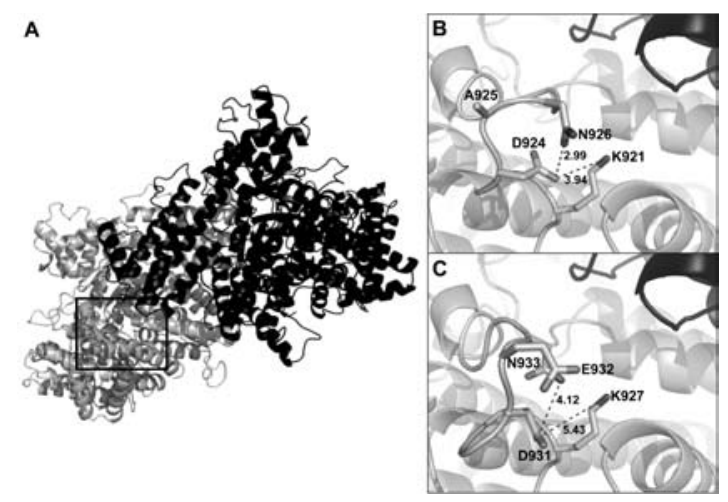

Fig. 4. Homology models constructed on the basis of the crystal coordinates of ZmPEPC-C4 (PDB accession code 1JQO). (A) AhPEPC-C4 dimer displayed using ribbons. Although the enzyme is tetrameric, only one of the dimers is shown for clarity of the figure. (B) The putative Gly binding site in the AhPEPC-C4 model, showing the residues that form the flexible region of the loop and the main interactions between them. (C) The putative Gly binding site in the ZmPEPC-C4 model showing the residues equivalent to those of the AhPEPC-C4 enzyme showed in panel (B). Distances are in angstroms.

of the side-chain of N926 (AhPEPC-C4 numbering) (Figure 4B). The electrostatic interaction between the conserved lysyl and aspartyl residues observed in the AhPEPC-C4 model may be important because it ensures that the two residues are oppositely charged and thus able to interact, also by means of saline bridges, with the charged $\alpha$-carboxyl and $\alpha$-amino groups, respectively, of the activator amino acid molecule. Although there is a considerable degree of uncertainty about the interactions observed in homology models, particularly in the case of this loop since there is no electronic density associated to it in the crystal structure used as a template to built the models, we believe that the interaction between these two opposite charged residues may be relevant for Gly binding, not only in the dicot PEPC-C4 enzymes but also in the monocot enzymes, since the residues involved are highly conserved among all PEPC-C4 (Fig. 3). A rigid docking of glycine in this position (not shown) suggested the feasibility of binding of the activator to these residues, as we propose. In fact, the mutation of K927 to a glutamine indicated the importance of this positively charged residue for Gly binding in ZmPEPC-C4 [28]. But in ZmPEPC-C4, and the other monocot enzymes, the influence of a nearby glutamic residue (E932, ZmPEPC-C4 numbering) that occupies the position equivalent to a neutral amino acid in the dicot enzymes, A925 in AhPEPC-C4, could either increase the $\mathrm{p} K_{\mathrm{a}}$ of the side-chain carboxyl group of D931 (ZmPEPC-C4 numbering), so that it would be protonated at physiological $\mathrm{pH}$, and/or exert electrostatic repulsion so that the carboxyl group of D931 moves from the position observed in the homology model of AhPEPC-C4. This was in fact observed in the model of the maize enzyme, where the position of D931 is significantly different from that observed in the AhPEPC-C4 model and consistent with an electrostatic repulsion between the two negatively charged residues (Fig. 4C). As a consequence of this, D931 and K927 in the maize enzyme model are not as well positioned to bind the activator molecule as they are in the amaranth enzyme 
model, as indicated by a rigid docking of the Gly molecule in this site (not shown), which is consistent with the $A_{0.5}$ for Gly being much higher in ZmPEPC-C4 than in AhPEPC-C4 (this work). This is also consistent with the experimental observation in ZmPEPC-C4 that the E932Q mutant exhibits an increased affinity for Gly [28]. The closeness of E932 to D931 would hinder the binding of the amino acid activator, but still the activator could bind if there were a ligand-induced conformational change, as in a typical induced fit process. Binding of the activator would therefore require a conformational change of the ZmPEPC-C4 but not of the AhPEPC-C4 enzyme, consistent not only with the observed differences in Gly affinity between the two kinds of PEPC-C4 isoenzymes but also with the triggering of the allosteric transition that follows the binding of Gly in the maize enzyme and that leads to its activation. This is because conformational changes in the putative Gly-binding loop would propagate to the whole quaternary tetrameric structure, given its proximity to the dimer-dimer interface. The allosteric transition would not occur in the amaranth enzyme, thus accounting for the huge differences between the amaranth and the maize enzymes in their degree of activation achieved at saturation by Gly.

\section{Conclusions}

The differences in the kinetic features of PEPC-C4 isoenzymes from monocot and dicot plants extend beyond the differences in their sensitivity to Gly already known. Both types of isoenzymes also differ in their affinity for the substrate PEP, the activator Glc6P and the inhibitor malate. The lack of activation by Gly of the dicot isoenzymes is mainly compensated by their higher affinity for the substrate PEP and their lower affinity for the inhibitor malate than those exhibited by the monocot isoenzymes. Two conserved and oppositely charged residues are likely important for binding of the $\alpha$-carboxyl and $\alpha$-amine groups of Gly in both kinds of PEPC-C4 enzymes, whereas a nearby non-conserved residue, which is neutral in dicot enzymes and negatively charged in monocot enzymes, may be responsible for differences between them in the conformation of the loop forming the Gly binding-site, and in turn, for their differences in Gly sensitivity. In the monocot enzymes it appears that this loop would undergo an activator-induced conformational change, thus accounting for both the poor affinity for the activator and the high degree of activation exhibited by the enzymes from monocot plants such as maize. Clearly, to support our proposal, the crystal structure of both kind of PEPC-C4 isoenzymes in complex with Gly must be determined, a task that is currently under way in our laboratory.

\section{Experimental}

\section{Chemicals and biochemicals}

PEP (monocyclohexylammonium salt), $\beta$-NADH (disodium salt), porcine heart malic dehydrogenase, chymostatin, Glc6P (monosodium salt), glycine, PMSF (phenylmethanesulfonyl fluoride), HEPES (4-(2-Hydroxyethyl)piperazine-1-ethanesulfonic acid, $\mathrm{N}-\left(2\right.$-Hydroxyethyl)piperazine- $\mathrm{N}^{\prime}-(2$-ethanesulfonic acid), and $\beta$-mercaptoethanol were purchased from Sigma-Aldrich. EDTA (ethylenediaminetetraacetic acid) (disodium salt) was from Merck. All other chemicals of analytical grade were from standard suppliers.

\section{Plant material}

Plants of maize (Zea mays L., cv. Chalqueño) were raised in soil culture in a naturally illuminated greenhouse at temperature between 20 and $30{ }^{\circ} \mathrm{C}$ and $12 \mathrm{~h}$ photoperiod. Fully expanded leaves were used for the experiments. Amaranth (Amaranthus hypochondriacus L.) leaves were purchased in local markets.

\section{Phosphoenolpyruvate carboxylase extraction, purification and assay}

Plants were kept in darkness for at least $6 \mathrm{~h}$ prior to extraction. Leaf segments $(50 \mathrm{~g})$ were extracted at $4{ }^{\circ} \mathrm{C}$ using a Waring Blendor, in a tissue:buffer ratio of 5:1 (w/v), using 50 $\mathrm{mM}$ HEPES-KOH buffer, $\mathrm{pH}$ 7.4, containing $1 \mathrm{mM}$ EDTA, $100 \mu \mathrm{g} / \mathrm{ml}$ chymostatine, $1 \mathrm{mM}$ PMSF and $10 \mathrm{mM} \beta$-mercaptoethanol. Both enzymes were purified as nontruncated and nonphosphorylated, night-forms, as described elsewhere [31]. By the criterion of SDS-PAGE (sodium dodecyl sulfate polyacrylamide gel electrophoresis), the enzyme preparations used in this study were more than 95\% homogeneous for ZmPEPC$\mathrm{C} 4$ and $\approx 60 \%$ homogeneous for AhPEPC-C4. Conventional SDS-PAGE was run on $7.5 \%$ polyacrylamide minislab gels after Laemmli [32]. Protein was measured by the method of Bradford [33], using bovine serum albumin as the standard. The final specific activities of the enzyme preparations used, determined in a standard assay, were $37 \mathrm{U} / \mathrm{mg}$ protein for $\mathrm{Zm}$ PEPC-C4 and $9 \mathrm{U} / \mathrm{mg}$ protein for AhPEPC-C4.

\section{Phosphoenolpyruvate carboxylase assay and kinetic studies}

PEPC activity was assayed spectrophotometrically at $30{ }^{\circ} \mathrm{C}$ in a coupled enzymatic assay by monitoring NADH oxidation at $340 \mathrm{~nm}\left(\varepsilon=6,220 \mathrm{M}^{-1} \mathrm{~cm}^{-1}\right)$ with a Lambda Bio 10 (Perkin Elmer) UV/VIS spectrophotometer equipped with a kinetics software package and thermostated cell. The standard assay medium, final volume of $0.5 \mathrm{~mL}$, consisted of $100 \mathrm{mM}$ HEPES-KOH buffer, pH 7.3, containing $10 \mathrm{mM} \mathrm{NaHCO}_{3}$, $1 \mathrm{mM}$ EDTA, five units of malate dehydrogenase, $0.2 \mathrm{mM}$ NADH, $5 \mathrm{mM}$ total PEP (tPEP) and $10 \mathrm{mM} \mathrm{MgCl}_{2}$. The $\mathrm{pH}$ of the PEP stock solution was previously adjusted to the $\mathrm{pH}$ of the assay. The reaction was started by addition of the enzyme preparation. Rates in the absence of PEP were negligible. PEP was stable in the assay medium in the presence of the enzyme preparation but absence of $\mathrm{Mg}^{2+}$ ions or bicarbonate. Each determination was performed at least in duplicate. One unit of PEPC is defined as the amount of enzyme needed to catalyze 
the formation of $1 \mu \mathrm{mol}$ of oxalacetate per min under our experimental conditions.

Steady-state initial velocity studies were performed under the same assay conditions than the standard assay described above, but using the concentrations of tPEP, malate, Glc6P or Gly stated in each experiment. Given that the preferred substrate of the PEPC-C4 isoenzymes is the complex between the $\mathrm{Mg}^{2+}$ ion and the substrate PEP $[2,3,34]$, we calculate the amounts of total magnesium (added at the assay media as $\mathrm{MgCl}_{2}$ ) and tPEP required to keep the concentration of $\mathrm{fMg}^{2+}$ at $0.4 \mathrm{mM}$, which is the concentration estimated to exist in the plant cytosol [18]. For this aim, we used the procedure and dissociation constants of MgPEP, MgGlc6P, and MgGly complexes described elsewhere [3,14]. No exogenous bicarbonate was added to the assay media, so that the concentration of bicarbonate was $0.1 \mathrm{mM}$ [14]. We display the results of the kinetics of saturation of the enzyme by its substrate PEP by considering tPEP as the variable substrate, instead of MgPEP, to facilitate the evaluation of the data in the physiological range of concentration of this metabolite. The $I_{50}$ for malate and the $A_{0.5}$ for Glc6P or Gly were determined at $\mathrm{pH} 7.3$ as described in [14], using $0.4 \mathrm{mM} \mathrm{fMg}^{2+}$ and $0.1 \mathrm{mM} \mathrm{NaHCO}_{3}$ but $0.2 \mathrm{mM}$ tPEP. Malate concentrations ranged from 0 to $20 \mathrm{mM}$; Glc6P concentrations from 0 to $20 \mathrm{mM}$; and Gly concentrations from 0 to $120 \mathrm{mM}$ in the absence of malate, or from 0 to $325 \mathrm{mM}$ in the presence of this inhibitor.

\section{Data analysis}

Kinetic data were analyzed by nonlinear regression calculations using a commercial computing program formulated with the algorithm of Marquardt [35]. Initial velocity data depending upon varied concentration of substrate were fitted to a Hill equation (equation 1):

$$
v / V_{\max }=[\mathrm{S}]^{h} /\left(S_{0.5^{h}}+[\mathrm{S}]^{h}\right)
$$

where $v$ is the experimentally determined initial velocity, $V_{\max }$ the maximum velocity, $[\mathrm{S}]$ the concentration of the variable substrate, $S_{0.5}$ the concentration of substrate that gives halfmaximum velocity, and $h$ the Hill coefficient.

In the experiments in which the concentration of the activator was varied at constant concentration of substrates, equation 2 was used:

$$
\left.v_{\mathrm{a}} / v_{0}=\operatorname{Act}_{\max }[\mathrm{A}]^{h} /\left(A_{0.5}{ }^{h}+[\mathrm{A}]^{h}\right)\right\}+1,
$$

where $v \mathrm{a}$ and $v_{\mathrm{o}}$ are the initial velocities in the presence and absence of activator, respectively, $A c t_{\max }$ is the maximum activation obtained at saturating activator concentrations, $[\mathrm{A}]$ is the activator concentration, and $A_{0.5}$ is the concentration of activator that gives half-maximum activation at fixed concentrations of substrates.

When the concentration of inhibitor was varied at constant concentration of substrates, the experimental data were fitted to equation 3 :

$$
v_{\mathrm{i}} / v_{\mathrm{o}}=I_{50} /\left(I_{50}+[\mathrm{I}]\right)
$$

where $v i$ and $v_{\mathrm{o}}$ are the initial velocities in the presence and absence of inhibitor, respectively, $I_{50}$ is the concentration of inhibitor that gives half-maximum inhibition at fixed concentrations of substrates, and [I] is the inhibitor concentration.

The points in the figures are the experimentally determined values, whereas the curves are calculated from fits of these data to the appropriate equation. The best fits were determined by the relative fit error, error of the constants and absence of significant correlation between the residuals, and other relevant variables like observed velocities, substrate concentration and data number.

\section{Sequence alignments and homology model building}

The search for PEPC-C4 sequences was performed at the World Wide Web site of the National Center for Biotechnology Information (NCBI) (http://www.ncbi.nlm.nih.gov) [36]. The integrated database retrieval system ENTREZ [37] was used to access the NCBI database. Progressive multiple sequence alignment was carried out with the Clustal_X package [38], using penalties based on secondary structure.

The three-dimensional homology models of AhPEPC-C4 and ZmPEPC-C4 were built on the basis of the crystal coordinates of ZmPEPC-C4 (PDB accesion code 1JQO) [17], which is $76.6 \%$ identical to AhPEPC-C4 at the amino acid level. The models were constructed using the automatic protein structure homology-modeling server SWISS-MODEL [39] (http://www. expasy.org/swissmod/). The same solution was always obtained after repeated submissions of the data to this server. The models were validated using ProCheck [40]. The figure was created with PyMOL [41].

\section{Acknowledgments}

We acknowledge the financial support of the Dirección General de Apoyo al Personal Académico (DGAPA) of the National Autonomous University of Mexico (UNAM) (PAPIIT grant IN216911) to RAMC. RGT is a recipient of a scholarship from the National Council of Science and Technology (CONACYT), México.

\section{References}

1. Hatch, M. D. Plant Cell Physiol. 1992, 33, 333-342.

2. Rodríguez-Sotres, R.; Muñoz-Clares, R. A. Arch. Biochem. Biophys. 1990, 276, 180-190.

3. Tovar-Méndez, A.; Rodríguez-Sotres, R.; López-Valentín, D. M.; Muñoz-Clares, R. A. Biochem. J. 1998, 332, 633-642.

4. Coombs, J.; Baldry, C. W.; Bucke, C. Biochem. J. 1972, 130, $25 \mathrm{P}$.

5. Wong, K. F.; Davies, D. D. Biochem. J. 1973, 131, 451-458.

6. Bandarian, V.; Poehner, W. J.; Grover, S. D. Plant Physiol. 1992, 100, 1411-1416. 
7. Doncaster, H. D.; Leegood, R. C. Plant Physiol. 1987, 84, 8287.

8. Mújica-Jiménez, C.; Castellanos-Martínez, A.; Muñoz-Clares, R. A. Biochim. Biophys. Acta 1998, 1386, 132-144.

9. Nishikido, T; Takanashi, H. Biochem. Biophys. Res. Commun. 1973, 53, 126-133.

10. Huber, S. C.; Edwards, G. E. Plant Physiol. 1975, 56, 324-331.

11. Jiao, J.; Chollet, R. Arch. Biochem. Biophys. 1988, 261, 409417.

12. Echevarría, C.; Pacquit, V.; Bakrim, N.; Osuna, L.; Delgado, B.; Arrio-Dupont, M.; Vidal, J. Arch. Biochem. Biophys. 1994, 315, 425-430.

13. Duff, S. M. G.; Andreo, C. S.; Pacquit, V.; Lepiniec, L.; Sarath, G.; Condon, S. A.; Vidal, J.; Gadal, P.; Chollet, R. Eur. J. Biochem. 1995, 228, 92-95.

14. Tovar-Méndez, A.; Mújica-Jiménez, C.; Muñoz-Clares, R. Plant. Physiol. 2000, 123, 149-160.

15. Leegood, R. C.; von Caemmerer, S. Planta 1994, 192, 232-238.

16. Rodríguez-Sotres, R.; Muñoz-Clares, R. A. J. Plant Physiol. 1987, 128, 361-369.

17. Matsumura, H.; Xie, Y.; Shirakata, S.; Inoue, T.; Yoshinaga, T.; Ueno, Y.; Izui, K.; Kai, Y. Structure 2002, 10, 1721-1730.

18. Yazaki, Y.; Asukagawa, N.; Ishikawa, Y.; Ohta, E.; Sakata, M. Plant Cell Physiol. 1988, 29, 919-924

19. Jenkins, C. L. D.; Furbank, R. T.; Hatch, M.D. Plant Physiol. 1989, 91, 1372-1381.

20. Rajagopalan, A. V.; Tirumala Devi, M.; Raghavendra, A. S. Photosynth Res. 1993, 38, 51-60.

21. Huber, S. C.; Sugiyama, T. Plant Physiol. 1986, 81, 674-677.

22. Leegood, R. C. Planta 1985, 164, 163-171.

23. Stitt, M.; Heldt, H. W. Plant Physiol. 1985, 79, 599-608.

24. Wedding, R. T.; Black, M. K.; Meyer, C. R. Plant Physiol. 1990, 92, 456-461.

25. Takahashi-Terada, A.; Kotera, M.; Ohshima, K.; Furumoto, T.; Matsumura, H.; Kai, Y.; Izui, K. J. Biol. Chem. 2005, 280, 1179811806.
26. Yuan, J.; Sayegh, J.; Mendez, J.; Sward, L.; Sanches, N.; Sanchez, S.; Waldrop, G.; Grover, S. Photosyn. Res. 2006, 88, 73-81.

27. Endo, T.; Mihara, Y.; Furumoto, T.; Matsumura, H.; Kai, Y.; Izui, K. J. Exp. Bot. 2008, 59, 1811-1818.

28. Gonzalez, L.; Sanchez, S.; Horne, J.; Kanzaki, G.; Grover, S. FASEB J. 2007, 21, 806.3.

29. Kai, Y.; Matsumura, H.; Inoue, T.; Terada, K.; Nagara, Y.; Yoshinaga, T.; Kihara, A.; Tsumura, K.; Izui, K. Proc. Nat. Acad. Sci. 1999, 96, 823-828.

30. Matsumura, H.; Terada, M.; Shirakata, S.; Inoue, T.; Yoshinaga, T.; Izui, K.; Kai, Y. FEBS Lett. 1999, 458, 93-96.

31. Tovar-Méndez, A.; Mújica-Jiménez, C.; Muñoz-Clares, R. A. Biochim. Biophys. Acta 1997, 1337, 207-216.

32. Laemmli, U. K. Nature 1970, 227, 680.

33. Bradford, M. M. Anal. Biochem. 1976, 72, 248-254.

34. Wedding, R. T.; Rustin, P.; Meyer, C. R.; Black M. K. Plant Physiol. 1988, 88, 976-979.

35. Marquardt, D.W. J. Soc. Ind. Appl. Math. 1963, 11, 431-441.

36. Wheeler, D. L.; Barrett, T.; Benson, D. A.; Bryant, S. H.; Canese, K.; Chetvernin, V.; Church, D. M.; DiCuccio, M.; Edgar, R.; Federhen, S.; Geer, L. Y.; Helmberg, W.; Kapustin, Y.; Kenton, D. L.; Khovayko, O.; Lipman, D. J.; Madden, T. L.; Maglott, D. R.; Ostell, J.; Pruitt, K. D.; Schuler, G. D.; Schriml, L. M.; Sequeira, E.; Sherry, S. T.; Sirotkin, K.; Souvorov, A.; Starchenko, G.; Suzek, T. O.; Tatusov, R.; Tatusova, T. A.; Wagner, L.; Yaschenko, E. Nucleic Acids Res. 2006, 34, D173D180.

37. Geer, R. C.; Sayers, E. W. Brief Bioinform. 2003, 4, 179-184.

38. Thompson, J. D.; Gibson, T. J.; Plewniak, F.; Jeanmougin, F.; Higgins, D. G. Nucleic Acids Res. 1997, 25, 4876-4882.

39. Schwede, T.; Kopp, J.; Guex, N.; Peitsch, M. C. Nucleic Acids Res. 2003, 31, 3381-3385.

40. Laskowski, R. A.; MacArthur, M. W.; Moss, D.; Thornton, J.M. J. Appl. Cryst. 1993, 26, 283-291.

41. DeLano, W. L. 2002, PyMOL (DeLano Scientific, San Carlos, CA) http://www.pymol.org/. 